\title{
Effects of Methylphenidate on Sleep Functioning in Children with Attention-Deficit/Hyperactivity Disorder
}

\author{
Stephen P. Becker, Ph.D. ${ }^{1,2,4}$, Tanya E. Froehlich, M.D., M.S. ${ }^{2,3}$, and Jeffery N. Epstein, Ph.D. \\ 1,2 \\ ${ }^{1}$ Division of Behavioral Medicine and Clinical Psychology, Cincinnati Children's Hospital Medical \\ Center, Cincinnati, Ohio \\ ${ }^{2}$ Department of Pediatrics, University of Cincinnati College of Medicine, Cincinnati, Ohio \\ ${ }^{3}$ Division of Developmental and Behavioral Pediatrics, Cincinnati Children's Hospital Medical \\ Center, Cincinnati, Ohio
}

\begin{abstract}
Objective-To examine the effects of stimulant medication on the sleep functioning of children with attention-deficit/hyperactivity disorder (ADHD) and identify predictors of sleep problems as a side effect of taking stimulant medication.
\end{abstract}

\begin{abstract}
Method-One-hundred-sixty-three stimulant-naïve children (72\% male) ages 7-11 years diagnosed with ADHD (120 with ADHD Predominantly Inattentive Type, 43 with ADHD Combined Type) participated in a four-week, randomized, double-blind, placebo-controlled trial of once-daily (long-acting) methylphenidate (MPH). Parents completed weekly side effect ratings including an item related to sleep problems.
\end{abstract}

Results-Ten percent of patients had parent-rated sleep problems prior to the initiation of medication. Rates of parent-rated sleep problems during MPH titration generally increased with increasing MPH dose (placebo: 8\%; low dose: 18\%; medium dose: 15\%; high dose: 25\%). Differences emerged between children with $(n=16)$ or without $(n=147)$ pre-existing sleep problems. Although $23 \%$ of children without pre-existing sleep problems went on to have sleep problems at the highest MPH dose, only $37.5 \%$ of children with pre-existing sleep problems still had sleep problems at the highest MPH dose. Lower weight and lower body mass index (BMI) were associated with increased sleep problems during MPH titration.

Conclusion-This study demonstrated a general association between increased MPH dose and increased sleep problems in children with ADHD, particularly for children of lower weight/BMI. However, a substantial proportion of children with pre-existing sleep difficulties no longer had sleep problems on the highest MPH dose, which may help explain mixed findings reported to date in studies examining the impact of MPH on sleep functioning in children with ADHD and suggests that MPH dose titration should not be avoided solely on the basis of a child's premorbid

\footnotetext{
${ }^{4}$ Address correspondence to: Stephen P. Becker, Ph.D., Division of Behavioral Medicine and Clinical Psychology, Cincinnati Children's Hospital Medical Center, 3333 Burnet Avenue MLC 10006, Cincinnati, Ohio, 45229, USA. ; Email: stephen.becker@cchmc.org. Telephone: +1 (513) 803-2066. Fax: +1 (513) 803-0084

Conflicts of Interest: None of the authors have any conflicts of interest.
} 
sleep problems. Future research is needed to replicate and extend these findings to more specific domains of sleep functioning and to identify differences between children with persistent or improved sleep functioning as a result of MPH use.

\section{Keywords}

attention-deficit/hyperactivity disorder; medication; methylphenidate; OROS MPH (Concerta $\left.{ }^{\circledR}\right)$; side effects; sleep; stimulant medication

\section{Introduction}

Children with attention-deficit/hyperactivity disorder (ADHD), whether taking stimulant medications or stimulant-naïve, experience more sleep problems than their peers, including longer sleep onset, greater daytime sleepiness, and higher bedtime resistance ${ }^{1.3}$. Since the most common treatment for pediatric ADHD is stimulant medication ${ }^{4}, 5$, there has been long-standing interest in whether stimulant medication use complicates sleep problems in children with ADHD and, in particular, contributes to a delayed sleep onset latency ${ }^{6}$. This concern was first noted almost four decades ago, when Barkley's ${ }^{7}$ systematic review of the literature found sleep problems to be the most frequently endorsed side effect experienced by children who were hyperactive and taking stimulant medication. Despite ongoing interest in the associations between stimulant use and sleep in children with $\mathrm{ADHD}^{6},{ }^{8}$, there are several limitations to the extant research in this area. For example, much of the research examining the impact of stimulant medication use on sleep was conducted using short-acting stimulant medications ${ }^{9-14}$, which have different pharmacodynamic and pharmacokinetic effects than today's commonly-used long-acting formulations. Moreover, previous research on this topic includes studies with small samples ${ }^{11}, 15,16$, lack of randomized designs ${ }^{16} 21$, and comparisons of active medications to each other ${ }^{22}, 23$.

To date, two open-label and three randomized, placebo-controlled studies have evaluated the impact of once-daily methylphenidate (MPH) on the sleep functioning of children with ADHD. Unfortunately, the findings across these studies are quite mixed. The two open-label studies both found that sleep problems were the most frequently-endorsed side effect while taking MPH. Specifically, in a sample of 24 children (ages 6-12 years; 92\% male) with ADHD (67\% diagnosed with ADHD Combined Type [ADHD-C]), Kim et al. ${ }^{16}$ found that $25 \%$ experienced insomnia as reported by parents on the "insomnia or trouble sleeping" item on the Barkley Side Effect Rating Scale (BSERS). Similarly, Wilens et al. ${ }^{20}$ found that $20 \%$ of children (ages 6-13; 83\% male) with ADHD (76\% with ADHD-C) had medicationrelated insomnia as reported by parents during clinical visits (a systematic scale to assess for adverse events was not used). Mixed findings were reported in the RCT studies. Faraone and colleagues $^{22}$ found that osmotic-release oral system (OROS) MPH did not increase parentrated sleep problems on the Children's Sleep Habits Questionnaire in a sample of 89 children (ages 6-12) with ADHD, and Swanson et al. ${ }^{23}$ found rates of parent-reported "insomnia or trouble sleeping" on the BSERS among children (ages 6-12; 74\% male) with ADHD (82\% with ADHD-C) to be higher on placebo than on active OROS MPH. In contrast, Stein et al. ${ }^{15}$ also used the "insomnia or trouble sleeping" item on the parent-reported BSERS and found a dose-response relation between MPH and sleep problems in 47 children (ages 5-16 
years; 70\% male) with ADHD (68\% with ADHD-C) whereby sleep problems increased linearly with increasing MPH dose. Given the conflicting findings across these studies to date, additional studies are needed.

Furthermore, since there is conflicting evidence regarding the impact of MPH on the sleep functioning of children with $\mathrm{ADHD}^{15},{ }^{22},{ }^{23}$, it is important to evaluate the role of preexisting sleep functioning. It is possible that children who experience sleep side effects while taking medication had pre-existing sleep difficulties that simply persist with medication use. In line with this possibility, pre-existing sleep problems have been found to significantly predict sleep problems during MPH titration (i.e., administration of different doses that are carefully monitored for side effects and response) ${ }^{16,22}$. However, improvement in sleep functioning after initiating medication use is also possible. For instance, Faraone et al. ${ }^{22}$ did not find MPH to impact the sleep functioning of children with ADHD and acknowledged that "it is possible that sleep problems occurred for some children while decreasing for others" 22,p.313. However, we are unaware of any study that has directly examined the extent to which children with ADHD experience worsened or improved sleep after initiating stimulant medication use compared to their pre-medication baseline.

Finally, in addition to evaluating the impact of MPH on the sleep functioning of children with ADHD, it is important to determine whether demographic or other child characteristics predict sleep functioning during MPH titration. Such information would be helpful to pediatricians and other prescribers in determining which children may experience sleep side effects after starting MPH and be able to help those families prepare and/or problem solve around sleep problems during MPH titration. In previous research, younger age and lower weight have been found to be associated with stimulant-related sleep problems among children with $\mathrm{ADHD}^{15},{ }^{16}$, although it is important to note that exceptions have also been reported $^{22}$. Comorbid internalizing symptoms and ADHD symptom severity may also be associated with stimulant-related sleep problems ${ }^{18}$.

The goal of this study is to examine the effects of once-daily (long-acting) MPH on the sleep functioning of children with ADHD using a randomized, double-blind, placebo-controlled trial. In addition to evaluating whether increased MPH dose was associated with increased sleep problems, we evaluated the effects of premorbid sleep problems on MPH-related sleep problems. Finally, we explored whether certain demographic (e.g., age, sex, socio-economic status [SES]) or other child characteristics (e.g., weight, body mass index [BMI], comorbidity) predict sleep problems during MPH titration.

\section{Methods}

\section{Participants}

Participants were 163 children (117 boys) recruited for a study examining the effects of MPH on the functioning of stimulant-naïve children with ADHD between the ages of 7 and 11 years $(M=8.41, S D=1.24)$. The children were $82 \%$ White, $12 \%$ Black, $4 \%$ Hispanic, and $2 \%$ other. The medication trial from which data for the present study were drawn oversampled for children with ADHD Predominantly Inattentive Type (ADHD-I) in order to examine medication response in children with ADHD-I specifically since this is the most 
common subtype in population-based studies ${ }^{24}$ and children with ADHD-I have been understudied in research examining sleep and $\mathrm{ADHD}^{25}$; children with ADHD

Predominantly Hyperactive-Impulsive Type were excluded. Per criteria described below, 120 children were diagnosed with ADHD-I and 43 children were diagnosed with ADHD-C. Twenty-eight percent of the children $(n=45)$ met criteria for a disruptive behavior disorder (i.e., oppositional defiant disorder [ODD] or conduct disorder [CD]), 32\% $(n=52)$ met criteria for an anxiety disorder (i.e., generalized anxiety, social phobia, separation anxiety, specific phobia, panic disorder, agoraphobia, obsessive-compulsive, or post-traumatic stress), and $1 \%(n=2)$ met criteria for a depressive disorder (i.e., dysthymia or major depressive disorder).

\section{Procedures}

Recruitment and diagnostic procedures have been described elsewhere ${ }^{26}, 27$ and are briefly described here. Written informed consent/assent was obtained from all parents/caregivers and participants according to the IRB-approved protocol. ADHD diagnosis was determined using methodology similar to the Multimodal Treatment Study of ADHD. Children were considered to have met the criteria for a symptom domain (i.e., inattention and/or hyperactivity/impulsivity) if the parent/caregiver on the Diagnostic Interview Schedule for Children-Version IV-Parent Report (DISC-IV-P) ${ }^{28}$ and the teacher on the Vanderbilt ADHD Teacher Rating Scale (VADPRS) ${ }^{29}$ reported six non-overlapping symptoms in a symptom domain and both the parent and the teacher reported at least four symptoms in that domain. Participants were required to meet ADHD DSM- $I V$ criteria for onset age, pervasiveness, and impairment as reported on the DISC-IV-P. In addition, participants were administered the Wechsler Abbreviated Scale of Intelligence and the Wechsler Individual Achievement Test Word Reading and Numerical Operations subtests and required to achieve standard scores of at least 80 on these measurements to exclude possible intellectual disability and/or learning disorders. Children were evaluated for psychiatric comorbidities using the DISC-P (described below). Children with mania/hypomania were excluded; comorbid ODD, CD, depression, and anxiety disorders were allowed if ADHD, rather than a comorbid disorder, was considered the primary cause of the child's impairment. Children were excluded if their medical history suggested significant brain injury.

\section{Medication Trial}

Subjects participated in a 4-week within-subject, randomized, double-blind, placebocontrolled crossover trial of long-acting OROS MPH (Concerta ${ }^{\circledR}$, Janssen Pharmaceuticals, Inc., Titusville, NJ), during which children were randomly assigned to one of six dosing schedules (see Froehlich et al. ${ }^{27}$ for exact dosing schedules) that included three active dosage weeks $(18,27$, or $36 \mathrm{mg}$ for children $\leq 25 \mathrm{~kg}[n=31]$; 18,36 , or $54 \mathrm{mg}$ for children $>25 \mathrm{~kg}[n=132])$ and one week of placebo. Of note, the dosages used in this study are FDA-approved for use with children $\Varangle 6$ years old and are identical to the doses examined in other trials examining the impact of OROS MPH on sleep ${ }^{15}, 22,23$. The randomization schemes were randomly selected with the following two rules in place: (1) children could not begin Week 1 of the medication trial on anything higher than 18mg, and (2) children could not go from placebo to $54 \mathrm{mg}$ on consecutive weeks. Study medication consisted of identical capsules filled with an inert white powder (placebo) or the prescribed dose of 
Concerta ${ }^{\circledR}$ over-encapsulated to preserve the double-blind design. At the end of each week, parents completed the VADPRS ${ }^{30}$ and Pittsburgh Side Effects Rating Scale (PSERS) ${ }^{31}$ (described below).

\section{Measures}

Diagnostic Interview Schedule for Children, Version IV, Parent Version (DISCIV-P)—The DISC-IV-P ${ }^{28}$ was administered to parents at baseline to assist in assessing $D S M-I V$ criteria for ADHD as well as comorbid disruptive behavior, anxiety, and mood diagnoses. The DISC-IV-P is a structured diagnostic interview designed for use in epidemiological and clinical studies. It contains algorithms to generate $D S M-I V$-based diagnoses and has demonstrated excellent reliability and validity ${ }^{28}$.

Vanderbilt ADHD Diagnostic Parent Rating Scale (VADPRS)—The VADPRS is a parent-report scale with good internal consistency, factor structure, and concurrent validity for the assessment of $\mathrm{ADHD}^{30}$. The VADPRS includes the 18 DSM-IV ADHD symptoms rated on a 4-point scale that indicates how frequently each ADHD symptom occurs $(0=$ never, 1 = occasionally, 2 = often, $3=$ very often). In addition, the VADPRS includes ODD (8 items), CD (14 items), anxiety (3 items), and depression (4 items) comorbidity screening scales ${ }^{30},{ }^{32}$. Symptom scores at baseline were used in the present study (as $=.84$ for inattention, .92 for hyperactivity-impulsivity, .94 for ODD, .78 for $\mathrm{CD}, .80$ for anxiety, and . 90 for depression).

Pittsburgh Side Effects Rating Scale (PSERS)-The PSERS ${ }^{31}$ is a frequently-used measure of pre-existing problems and medication-related side effects. Each item is rated by the parent on a four-point scale indicating the severity of the problem over the past week ( 0 $=$ none, $1=$ mild, $2=$ moderate, $3=$ severe). The item assessing sleep problems ("trouble sleeping") completed by parents at baseline and each week during the medication trial was used in the current study.

\section{Analyses}

Frequency of pre-existing sleep problems was calculated to describe the sample. As in previous research examining medication-related side effects in children with $\mathrm{ADHD}^{10},{ }^{33}$, children with a rating of "none" or "mild" on the sleep problems item was classified as not having a pre-existing sleep problem, whereas children with a rating of "moderate" or "severe" were classified as having a pre-existing sleep problem. This same approach was used to determine the absence or presence of sleep problems at the placebo, low, medium, and high doses during the medication trial. Second, a repeated measures analysis of variance (ANOVA) using continuous sleep functioning scores at each of the five time-points (baseline, placebo, low dose, medium dose, high dose) was conducted to test whether there was a dose-response of sleep problem side effects with increasing MPH dose. A statistically significant ANOVA was followed up with post hoc tests using a Bonferroni correction.

Next, we evaluated the extent to which pre-existing sleep problems contributed to sleep problems during the medication trial. The role of pre-existing sleep problems was examined in two ways. First, repeated measures ANOVA was used to examine pre-existing sleep as a 
predictor of the dose-response relation between MPH and sleep problems. ANOVAs evaluating the continuous pre-existing sleep functioning score or the dichotomous presence/ absence of pre-existing sleep problems were conducted separately. Second, the dichotomous classification of participants having or not having sleep problems prior to starting MPH and on their highest medication dose was examined. A McNemar's modified $\chi^{2}$ test was used to test the odds of patients switching categories (i.e., having no pre-existing sleep problems to having MPH-related sleep problems and vice versa).

Finally, we conducted exploratory analyses to examine whether child characteristics/baseline variables moderated the dose-response relation between MPH and sleep problems. Repeated measures ANOVAs were conducted that included continuously-measured sleep problems at the four titration time-points (placebo, low dose, medium dose, high dose), the child characteristic/baseline variable as a between-subjects factor (dichotomous variables) or predictor covariate (continuous variables), and the interaction between sleep problems and the child characteristic/baseline variable. A separate repeated measures ANOVA was conducted for each of the following child characteristics/baseline variables: weight in $\mathrm{kg}$ (continuously measured), BMI, age, sex, race, SES (a dichotomous variable whereby low SES was defined as having parent-report of any of the following areas of public support: welfare, Medicaid, food stamps, or participation in the Special Supplemental Nutrition Program for Women, Infants, and Children [WIC]), ADHD subtype, presence of a comorbid disruptive behavior disorder (ODD/CD), presence of a comorbid anxiety disorder (only two children had a comorbid depressive disorder so this comorbidity was not considered), and continuous psychopathology symptom scores (i.e., inattention, hyperactivity-impulsivity, oppositional defiant behaviors, conduct disorder, anxiety, depression). Given the exploratory nature of these analyses, we did not correct for the number of analyses conducted. Statistically significant $(p<.05)$ interactions were graphed to examine the nature of the interaction effect.

\section{Missing Data}

Of the 171 children who participated in the medication trial, the sleep problems item was missing for four participants at baseline, two participants at placebo, two participants at low dose, six participants at medium dose, and three participants at high dose. In order to include as many of the participants as possible, a carry-forward approach was used for participants missing sleep problems data at the medium and high doses. Specifically, if the sleep problem score at the medium dose was missing the score from the low dose was used, and if the sleep problem score at the high dose was missing the score from the medium dose was used. Since a carry-forward approach would not be possible when data was missing at baseline, placebo, or the low dose, the eight children with data missing at one of these time-points were excluded from the study. These eight participants did not differ from the remaining 163 participants on age, sex, race, SES, weight, BMI, ADHD subtype, IQ, or academic achievement (all $p s>.05$ ). In addition, the nine participants with sleep data carried forward did not differ significantly from the 154 with complete sleep data on any of these variables (all $p \mathrm{~s}>.05$ ). Furthermore, the pattern of results was unchanged when the nine participants who had medium or high dose sleep data carried forward were excluded from the analyses. 


\section{Results}

\section{Titration Trial Medication Outcomes}

As noted above (see Missing Data), although all participants had a highest dose variable, the highest dose was not always $36 \mathrm{mg}$ for children $\leq 25 \mathrm{~kg}$ or $54 \mathrm{mg}$ for children $>25 \mathrm{~kg}$ (of note, age and weight category were only moderately correlated, $r=.38$ ). Specifically, the highest dose was $18 \mathrm{mg}$ for three participants (one child weighing $\leq 25 \mathrm{~kg}$ and two children weighing > $25 \mathrm{~kg}$ ), $36 \mathrm{mg}$ for 35 participants (this was highest dose possible for 30 children weighing $\leq 25 \mathrm{mg})$, and $54 \mathrm{mg}$ for 125 participants weighting $>25 \mathrm{mg}\left(M_{\mathrm{dose}}=49.47 \mathrm{mg}\right.$, $\left.S D_{\text {dose }}=8.57\right)$. The average $\mathrm{mg} / \mathrm{kg}$ was $1.53(S D=0.32$; range: $0.52-2.16)$.

\section{Effect of MPH Dose on Sleep Functioning in Children with ADHD}

Parents reported moderate or severe sleep problems prior to initiating the medication trial in 16 children $(9.8 \%$, with $9.2 \%$ having "moderate" sleep problems and $0.6 \%$ having "severe" sleep problems). Rates of sleep problems during the titration were $8.0 \%$ on placebo ( $7.4 \%$ moderate, $0.6 \%$ severe), $17.8 \%$ on low MPH dose (14.7\% moderate, $3.1 \%$ severe), $14.7 \%$ on medium MPH dose (10.4\% moderate, $4.3 \%$ severe), and $24.6 \%$ on high MPH dose (16.0\% moderate, $8.6 \%$ severe).

The repeated measures ANOVA indicated that sleep problems were significantly different across the five study time-points, $F(4,648)=18.74, p<.001, \eta_{\mathrm{p}}^{2}=.10$. As displayed in Figure 1, post- hoc tests indicated that pre-existing sleep functioning and sleep functioning while taking placebo did not differ from each other. However, both pre-existing and placebo sleep significantly differed from sleep functioning on all three of the MPH doses such that sleep problems increased with increasing MPH dosage (all $p s<.05$ ). Specifically, although sleep functioning on the low and medium doses were comparable $(p>0.05)$, children had more sleep problems on the high dose compared to the low and medium dose conditions ( $p$ s $<.05$; see Figure 1).

\section{The Role of Pre-Existing Sleep Functioning on MPH-Related Sleep Functioning}

Next, we examined the relationship between pre-existing sleep problems and MPH-related sleep problems. As summarized in Table 1, there was a statistically significant main effect (but no interaction effect) of pre-existing sleep problems on the dose-response relation between MPH and sleep problems when the continuous measure of pre-existing sleep problems was used, indicating that children with higher levels of pre-existing sleep problems had higher sleep problem ratings irrespective of medication dose. In addition, in the model examining the dichotomous presence of pre-existing sleep problems there was a significant dose $\times$ sleep problems interaction $(p=.02)$. Children with pre-existing sleep problems had higher sleep problems scores on placebo $\left(t=-6.77, p<.001, \eta_{\mathrm{p}}^{2}=.22\right)$ and on the low dose $\left(t=-2.41, p=.02, \eta_{\mathrm{p}}^{2}=.04\right)$ than children without pre-existing sleep problems, but children with or without pre-existing sleep problems did not differ on their sleep problem scores on the medium or high doses ( $p s=.22$ and .20 , respectively).

Next, we examined the odds ratio of switching from being a child without (or with) preexisting sleep problems to being a child with (or without) sleep problems at the high dose of 
medication (McNemar's odds ratio of 3.40 (95\% CI: 1.64, 7.72), $p=.0005$ ). As shown in Figure 2, 23.1\% of the 147 children without pre-existing sleep problems went on to have sleep problems at the highest MPH dose. In addition, 37.5\% of those with pre-existing sleep problems continued to have sleep problems on their highest dosage of medicine. However, the other $62.5 \%$ of children with pre-existing sleep problems were no longer classified as having sleep problems (i.e., had shifted to "none" or "mild" sleep problems) at the highest dose.

\section{Child Characteristics and Baseline Predictors of MPH-Related Sleep Functioning}

Finally, repeated measures ANOVAs were conducted to examine whether child characteristics or baseline variables other than pre-existing sleep problems predicted the dose-response relation between MPH and sleep problems. Results are summarized in Table 1. There was neither a main effect nor a dose $\times$ predictor effect for age, sex, ADHD symptom severity, or psychiatric comorbidity (as measured by the presence of a comorbid diagnosis or continuous comorbidity scores). Main effects emerged for race and ADHD subtype: White children had higher sleep problem scores than non-White children $(p=.005)$ and children with ADHD-I had higher sleep problem scores than children with ADHD-C ( $p$ $=.04)$. Although significant interactions with dose were not found for these predictor variables, we nevertheless explored the nature of the main effects, particularly since previous research has not found greater sleep problems among children with ADHD-I as compared to children with ADHD-C ${ }^{33},{ }^{35}$. Follow-up independent samples $t$-tests indicated that White children had significantly greater sleep problems than non-White children on placebo, low dose, and medium dose $(p s<.05)$ but did not differ at baseline or on high dose $(p s>.05)$. Children with ADHD-I or ADHD-C did not differ in their baseline sleep functioning; the only time-point where there was a significant subtype difference was at the low dose, with children with ADHD-I having higher sleep problem scores than children with ADHD-C ( $p$ $=.01)$.

A significant dose $\times$ SES interaction effect was found (see Table 1). Children receiving public assistance did not differ from other children on their sleep problem scores on placebo, low dose, or medium dose ( $p s=.36, .40$, and .84 , respectively) but children on public assistance had lower sleep problem scores than other children on the high dose $(t=2.57, p$ $\left.=.01, \eta_{\mathrm{p}}^{2}=.04\right)$. Finally, significant dose $\times$ weight and dose $\times$ BMI interaction effects were also found. Although weight was unassociated with sleep problem scores on placebo ( $p=$. 31 ), weight was significantly negatively associated with sleep problem scores on the low dose $\left(t=-2.10, p=.04, \eta_{\mathrm{p}}^{2}=.03\right)$, medium dose $\left(t=-2.40, p=.02, \eta_{\mathrm{p}}^{2}=.03\right)$, and high dose $\left(t=-2.50, p=.01, \eta_{\mathrm{p}}^{2}=.04\right)$. A similar pattern was found for BMI, which was unassociated with sleep problem scores on placebo $(p=.07)$ but was significantly negatively associated with sleep problem scores on the low dose $\left(t=-2.61, p=.01, \eta_{\mathrm{p}}^{2}=.04\right)$, medium dose $\left(t=-2.11, p=.04, \eta_{\mathrm{p}}{ }^{2}=.03\right)$, and high dose $\left(t=-3.53, p=.001, \eta_{\mathrm{p}}{ }^{2}=.07\right)$. These results were obtained using children's continuously-measured weight and BMI, but for ease of presentation we graphed the weight interaction using the weight dichotomy used in the medication trial randomization scheme (i.e., $\leq 25 \mathrm{~kg}$ vs. $>25 \mathrm{~kg}$ ). As displayed in Figure 3, children weighing $\leq 25 \mathrm{~kg}$ experience an immediate increase in sleep problems starting at 
the low dose that persists at higher doses, whereas children weighing more than $25 \mathrm{~kg}$ experience a step-wise progression in sleep problem side effects.

\section{Discussion}

The present study contributes to this literature by using data from a randomized, doubleblind, placebo-controlled trial to examine the impact of once-daily MPH treatment on sleep functioning in a sample of children diagnosed with ADHD. Further, we examined sleep functioning during medication use as compared to both pre-existing and placebo sleep functioning, considered the role of pre-existing sleep problems on MPH-related sleep problems, and explored possible predictors and moderators of sleep problems during medication titration.

Rates of sleep problems across studies of children with ADHD vary widely, with estimates generally ranging from $25-70 \%^{2},{ }^{3}$. In the present sample, approximately $10 \%$ of children with ADHD had sleep problems prior to the initiation of medication. Although this prevalence rate is lower than some previous studies, it is important to note several previous studies examining sleep problems in children with ADHD included children who were taking medication, which may have inflated rates of sleep problems. Stein et al. ${ }^{18}$ found that $13 \%$ of unmedicated children with ADHD had moderate to severe sleep problems, a rate very similar to the $10 \%$ of children in the present study.

Clear evidence was found for a dose-response relation between MPH dose and increasing rates of sleep problems. This was true whether we used either a dichotomous or continuous measure of sleep problems. Rates of dichotomized sleep problems rose to $15-18 \%$ at low and medium MPH doses (differing significantly from rates at baseline and placebo but not from each other), and then climbed to $25 \%$ at the high MPH dose. Several other studies have also reported sleep problem side effects to be between 20 and $25 \%$ on $\mathrm{MPH}^{15},{ }^{16},{ }^{20}$. We found a similar stepwise worsening in sleep functioning as a result of higher medication dose when using continuous sleep problem scores: children had significantly more sleep problems at the highest dose in comparison to the medium or low doses, and in turn children had more sleep problems at the medium or low doses in comparison to no medication (placebo or pre-existing sleep functioning; see Figure 1). This dose-response finding aligns with results reported by Stein et al. ${ }^{15}$ in a smaller sample of 47 children with ADHD and is in contrast to the study by Faraone and colleagues ${ }^{22}$ which failed to find an association between MPH use and sleep functioning. However, Faraone et al. ${ }^{22}$ compared baseline sleep functioning to endpoint sleep functioning after dose optimization, which the authors note may have resulted in an attenuation of effects if sleep side effects contributed to dosage adjustments during titration. Finally, in contrast to Swanson et al. ${ }^{23}$, we found no evidence of greater sleep problems when children were taking placebo as opposed to active medication. Rather, we found that pre-existing and placebo sleep did not differ from each other, and both significantly differed from all three active medication doses. In the Swanson et al. study, the investigators recruited children who were already being treated with MPH and assigned a dose level according to the children's pre-existing daily dose, which may account for the different results we obtained in a sample of medication-naïve children who underwent a trial of three different MPH doses (in addition to placebo). Presumably, since 
the participants in the Swanson et al. study had previously chosen to continue taking MPH, they are likely among those who tolerated MPH sleep and other side effects well, and if our findings hold, some with pre-existing sleep problems may have improved in this regard on MPH. In this case, when MPH was replaced with placebo in Swanson et al.'s study, sleep problems could have worsened, which would in fact be compatible with our results.

Consistent with previous research ${ }^{16},{ }^{22}$, pre-existing sleep problem scores were associated with greater sleep problems during the medication titration. However, children with and without sleep problems only differed in their sleep functioning on placebo and low dose; no differences were observed on the medium and high doses. Further, despite finding a doseresponse effect at the group level, our findings indicate that there is heterogeneity in how MPH affects the sleep functioning of children with ADHD. This heterogeneity has perhaps contributed to the mixed findings to date reported in both randomized trials ${ }^{15}, 22,23$ and observational studies ${ }^{17},{ }^{25},{ }^{34}, 35$. Specifically, we found that almost one-quarter of the 147 children without pre-existing sleep problems went on to have sleep problems at the highest dose of MPH. In contrast, among the 16 children with pre-existing sleep problems, almost two-thirds no longer had sleep problems at the highest MPH dose. This suggests that while MPH use increases risk for sleep problems at the group level, a sizeable subset of children with ADHD who have pre-existing sleep problems may actual experience an improvement in their sleep functioning as a result of MPH treatment. This is an important finding that should be investigated further in subsequent research. In particular, studies with a larger number of children with pre-existing sleep problems would be informative for identifying differences between children with persistent or improved sleep functioning as a result of MPH use. It is also possible that for some children with ADHD, the effect of MPH extends until bedtime and has a beneficial impact on bedtime routines and overall sleep functioning. In any event, our findings underscore the importance of assessing sleep prior to initiating MPH and also suggest that clinicians should not avoid prescribing a higher dosage of MPH based on the sole fact that a child with ADHD has pre-existing sleep problems.

Finally, we sought to identify child characteristics and baseline variables that predict the dose-response of MPH use in relation to sleep problems. The majority of variables (i.e., age, sex, ADHD symptom severity, comorbid diagnoses/symptoms) were unrelated to MPHrelated sleep problems. A main effect of subtype was found whereby children with ADHD-I had higher sleep problem scores than children with ADHD-C, although follow-up tests indicated that the subtypes did not differ in their pre-existing sleep functioning and only differed significantly on the low dose of MPH. It should also be noted that our sample consisted disproportionately of children with ADHD-I (74\%). Intriguingly, a dose $\times$ SES interaction was also found, whereby children in families with greater financial strain had fewer sleep problems than other children, but only on the high dose of medication. There is some evidence that expectations surrounding bedtime behaviors and optimal child development may differ across socioeconomic and racial groups ${ }^{36}$. Still, these studies do not speak to why our SES finding was specific to the high dose of MPH, and given the exploratory nature of this analysis as well as the fact that only $15 \%$ of our sample met criteria for low SES, this finding warrants replication in future work in order to bolster confidence that this is not a chance finding. Last, weight significantly predicted the doseresponse of MPH and sleep problems. This finding aligns with previous work directly 
linking weight to sleep side effects ${ }^{15}$. In addition to finding that children of lower weight were at increased risk for experiencing sleep side effects after initiating MPH treatment, results using a dichotomized weight variable suggest this is especially likely at lower doses of medication, whereas all children with ADHD tend to experience elevated sleep problem scores on high doses of MPH regardless of weight. This, in addition to the fact that we compared children with and without sleep problems at the highest MPH dose and found that these two groups did not differ in $\mathrm{mg} / \mathrm{kg}$ at the highest dose $(p>.10)$, suggests that the association between increased sleep problems on MPH and lower weight is not merely due to children with lower weight experiencing higher $\mathrm{mg} / \mathrm{kg}$ doses.

Strengths of this study include its rigorous randomized, double-blind, placebo-controlled design, systematic diagnosis of ADHD and comorbid conditions, assessment of pre-existing sleep problems, and large sample size as compared to most other studies examining MPH effects on sleep. Nevertheless, several limitations are important to note. First, participants were able to experience large dose increases given the various dosing schemes used in the current study, although as described above participants could not start on the highest dose of medication and likewise could not go immediately from placebo from the highest dose. Second, the measure of sleep functioning was limited to a single parent-report item. This is consistent with most other studies examining sleep side effects resulting from MPH initiation, as well as the side effects scale in the widely-distributed American Academy of Pediatrics (AAP) ADHD Toolkit. Nevertheless, clinical practice for assessing sleep is nuanced and varied (e.g., clinicians often ask additional questions related to sleep functioning), and it would be informative for future studies to use well-validated measures that would allow for greater specificity in the sleep domains measured since MPH may have a greater impact on some sleep domains (e.g., sleep onset latency) than others (e.g., night wakings, parasomnias) ${ }^{18}$. It would also be informative to incorporate other methods such as daily sleep diaries or actigraphy that would allow for an evaluation of sleep problem frequency as well as sleep problem severity. Third, our results are limited to initial medication titration and did not examine sleep functioning during MPH maintenance or evaluate long-term sleep behaviors. There is some evidence that sleep problems spike when MPH is initiated but dissipate to some degree with longer MPH use ${ }^{6,}{ }^{37}$, but ongoing sleep problems while taking MPH have also been reported ${ }^{10}$. Fourth, while we examined many demographics and other characteristics as possible predictors of MPH-related sleep functioning, we were unable to examine some potentially important predictors such as structure surrounding bedtime, parenting behaviors, or metabolism of MPH. Fifth, the current study over-sampled for children with ADHD-I, and although this may be considered a strength since most previous studies in this area have included few children with ADHD-I even though this is the most common subtype in community- and population-based samples ${ }^{24}$, it is possible that this sampling strategy impacted the findings since several studies indicate that children with ADHD-C have more sleep problems than children with $\mathrm{ADHD}^{31} \mathrm{I}^{33}$. However, this seems unlikely since a significant subtype $\times$ dose interaction did not emerge in the repeated measures ANOVA analysis. Finally, we recruited medicationnaïve children for this study and most children were White, so the sample may not generalize to all children with ADHD or children who have prior experience with a range of ADHD medications. Furthermore, our study consisted of school-aged children and findings 
may not apply to older children; examining the impact of MPH on the sleep functioning of adolescents with ADHD is itself an important area for investigation since rates of sleep problems in the general population rise as children enter adolescence ${ }^{38}$.

In conclusion, this study demonstrates a general stepwise association between increased MPH dose and increased sleep problems in children with ADHD, with $25 \%$ of children experiencing clinically significant sleep problems at the highest dose of MPH. However, several factors moderating the adverse effects of increasing MPH dose on sleep were identified. Although children with pre-existing sleep problems (compared to those without) were at increased risk for sleep problems while taking low dose MPH, a sizeable percentage of children with pre-existing sleep problems no longer reported sleep problems with higher dose MPH treatment. In addition, children who are of lower weight are especially prone to experiencing MPH-related sleep side effects, with the negative impact of MPH observed at even low doses of medication. Future research is needed to replicate these findings and extend them to more specific domains of sleep functioning.

\section{Acknowledgments}

Dr. Becker is supported by award number K23MH108603 from the National Institute of Mental Health (NIMH). This research was supported by grants from the National Institutes of Health (NIH) to Dr. Froehlich (K23MH083881) and Dr. Epstein (R01MH074770; K24MH064478). The content is solely the responsibility of the authors and does not necessarily represent the official views of the National Institutes of Health (NIH).

\section{References}

1. Cortese S, Faraone SV, Konofal E, et al. Sleep in children with attention-deficit/hyperactivity disorder: Meta-analysis of subjective and objective studies. J Am Acad Child Adolesc Psychiatry. 2009; 48:894-908. [PubMed: 19625983]

2. Kirov R, Brand S. Sleep problems and their effect in ADHD. Expert Rev Neurother. 2014; 14:28799. [PubMed: 24491141]

3. Yoon SY, Jain U, Shapiro C. Sleep in attention-deficit/hyperactivity disorder in children and adults: past, present, and future. Sleep Med Rev. 2012; 16:371-88. [PubMed: 22033171]

4. Castle L, Aubert RE, Verbrugge RR, et al. Trends in medication treatment for ADHD. J Atten Disord. 2007; 10:335-42. [PubMed: 17449832]

5. Visser SN, Danielson ML, Bitsko RH, et al. Trends in the parent-report of health care providerdiagnosed and medicated attention-deficit/hyperactivity disorder: United States, 2003-2011. J Am Acad Child Adolesc Psychiatry. 2014; 53:34-46. e2. [PubMed: 24342384]

6. Stein MA, Weiss M, Hlavaty L. ADHD treatments, sleep, and sleep problems: complex associations. Neurotherapeutics. 2012; 9:509-17. [PubMed: 22718078]

7. Barkley RA. A review of stimulant drug research with hyperactive children. J Child Psychol Psychiatry. 1977; 18:137-65. [PubMed: 326801]

8. Weiss MD, Craig SG, Davies G, et al. New research on the complex interaction of sleep and ADHD. Current Sleep Medicine Reports. 2015:1-8. [PubMed: 26618103]

9. Firestone P, Musten LM, Pisterman S, et al. Short-term side effects of stimulant medication are increased in preschool children with attention-deficit/hyperactivity disorder: a double-blind placebocontrolled study. J Child Adolesc Psychopharmacol. 1998; 8:13-25. [PubMed: 9639076]

10. Wigal T, Greenhill L, Chuang S, et al. Safety and tolerability of methylphenidate in preschool children with ADHD. J Am Acad Child Adolesc Psychiatry. 2006; 45:1294-303. [PubMed: 17028508]

11. Stein MA, Blondis TA, Schnitzler ER, et al. Methylphenidate dosing: twice daily versus three times daily. Pediatrics. 1996; 98:748-56. [PubMed: 8885956] 
12. Sangal RB, Owens J, Allen AJ, et al. Effects of atomoxetine and methylphenidate on sleep in children with ADHD. Sleep. 2006; 29:1573-85. [PubMed: 17252888]

13. Efron D, Jarman F, Barker M. Side effects of methylphenidate and dexamphetamine in children with attention deficit hyperactivity disorder: a double-blind, crossover trial. Pediatrics. 1997; 100:662-6. [PubMed: 9310521]

14. Barkley RA, McMurray MB, Edelbrock CS, et al. Side effects of methylphenidate in children with attention deficit hyperactivity disorder: a systemic, placebo-controlled evaluation. Pediatrics. 1990; 86:184-92. [PubMed: 2196520]

15. Stein MA, Sarampote CS, Waldman ID, et al. A dose-response study of OROS methylphenidate in children with attention-deficit/hyperactivity disorder. Pediatrics. 2003; 112:e404. [PubMed: 14595084]

16. Kim HW, Yoon IY, Cho SC, et al. The effect of OROS methylphenidate on the sleep of children with attention-deficit/hyperactivity disorder. Int Clin Psychopharmacol. 2010; 25:107-15. [PubMed: 20093941]

17. Becker SP, Langberg JM, Evans SW. Sleep problems predict comorbid externalizing behaviors and depression in young adolescents with attention-deficit/hyperactivity disorder. Eur Child Adolesc Psychiatry. 2015; 24:897-907. [PubMed: 25359419]

18. Stein MA. Unravelling sleep problems in treated and untreated children with ADHD. J Child Adolesc Psychopharmacol. 1999; 9:157-68. [PubMed: 10521009]

19. O'Brien LM, Ivanenko A, Crabtree VM, et al. The effect of stimulants on sleep characteristics in children with attention deficit/hyperactivity disorder. Sleep Med. 2003; 4:309-16. [PubMed: 14592303]

20. Wilens T, McBurnett K, Stein M, et al. ADHD treatment with once-daily OROS methylphenidate: final results from a long-term open-label study. J Am Acad Child Adolesc Psychiatry. 2005; 44:1015-23. [PubMed: 16175106]

21. Wilens T, Pelham W, Stein M, et al. ADHD treatment with once-daily OROS methylphenidate: interim 12-month results from a long-term open-label study. J Am Acad Child Adolesc Psychiatry. 2003; 42:424-33. [PubMed: 12649629]

22. Faraone SV, Glatt SJ, Bukstein OG, et al. Effects of once-daily oral and transdermal methylphenidate on sleep behavior of children with ADHD. J Atten Disord. 2009; 12:308-15. [PubMed: 18400982]

23. Swanson JM, Wigal SB, Wigal T, et al. A comparison of once-daily extended-release methylphenidate formulations in children with attention-deficit/hyperactivity disorder in the laboratory school (the Comacs Study). Pediatrics. 2004; 113:e206-16. [PubMed: 14993578]

24. Willcutt EG. The prevalence of DSM-IV attention-deficit/hyperactivity disorder: a meta-analytic review. Neurotherapeutics. 2012; 9:490-9. [PubMed: 22976615]

25. Becker SP, Pfiffner L, Stein MA, et al. Sleep habits in children with attention-deficit/hyperactivity disorder predominantly inattentive type and associations with comorbid psychopathology symptoms. Sleep Med. 2015; advance online publication. doi: 10.1016/j.sleep.2015.11.011

26. Epstein JN, Brinkman WB, Froehlich T, et al. Effects of stimulant medication, incentives, and event rate on reaction time variability in children with ADHD. Neuropsychopharmacology. 2011; 36:1060-72. [PubMed: 21248722]

27. Froehlich TE, Epstein JN, Nick TG, et al. Pharmacogenetic predictors of methylphenidate doseresponse in attention-deficit/hyperactivity disorder. J Am Acad Child Adolesc Psychiatry. 2011; 50:1129-39. e2. [PubMed: 22024001]

28. Shaffer D, Fisher P, Lucas CP, et al. NIMH Diagnostic Interview Schedule for Children Version IV (NIMH DISC-IV): description, differences from previous versions, and reliability of some common diagnoses. J Am Acad Child Adolesc Psychiatry. 2000; 39:28-38. [PubMed: 10638065]

29. Wolraich ML, Bard DE, Neas B, et al. The psychometric properties of the Vanderbilt attentiondeficit hyperactivity disorder diagnostic teacher rating scale in a community population. J Dev Behav Pediatr. 2013; 34:83-93. [PubMed: 23363973]

30. Wolraich ML, Lambert W, Doffing MA, et al. Psychometric properties of the Vanderbilt ADHD diagnostic parent rating scale in a referred population. J Pediatr Psychol. 2003; 28:559-67. [PubMed: 14602846] 
31. Pelham WE. Pharmacotherapy for children with attention-deficit hyperactivity disorder. School Psychology Review. 1993; 22:199-227.

32. Becker SP, Langberg JM, Vaughn AJ, et al. Clinical utility of the Vanderbilt ADHD diagnostic parent rating scale comorbidity screening scales. J Dev Behav Pediatr. 2012; 33:221-8. [PubMed: 22343479]

33. Daviss WB, Patel NC, Robb AS, et al. Clonidine for attention-deficit/hyperactivity disorder: II. ECG changes and adverse events analysis. J Am Acad Child Adolesc Psychiatry. 2008; 47:189-98. [PubMed: 18182964]

34. Mayes SD, Calhoun SL, Bixler EO, et al. ADHD subtypes and comorbid anxiety, depression, and oppositional-defiant disorder: differences in sleep problems. J Pediatr Psychol. 2009; 34:328-37. [PubMed: 18676503]

35. Mick E, Biederman J, Jetton J, et al. Sleep disturbances associated with attention deficit hyperactivity disorder: the impact of psychiatric comorbidity and pharmacotherapy. J Child Adolesc Psychopharmacol. 2000; 10:223-31. [PubMed: 11052412]

36. Milan S, Snow S, Belay S. The context of preschool children's sleep: racial/ethnic differences in sleep locations, routines, and concerns. J Fam Psychol. 2007; 21:20-8. [PubMed: 17371106]

37. Findling RL, Bukstein OG, Melmed RD, et al. A randomized, double-blind, placebo-controlled, parallel-group study of methylphenidate transdermal system in pediatric patients with attentiondeficit/hyperactivity disorder. J Clin Psychiatry. 2008; 69:149-59. [PubMed: 18312050]

38. Becker SP, Langberg JM, Byars KC. Advancing a biopsychosocial and contextual model of sleep in adolescence: a review and introduction to the special issue. J Youth Adolesc. 2015; 44:239-70. [PubMed: 25552436] 


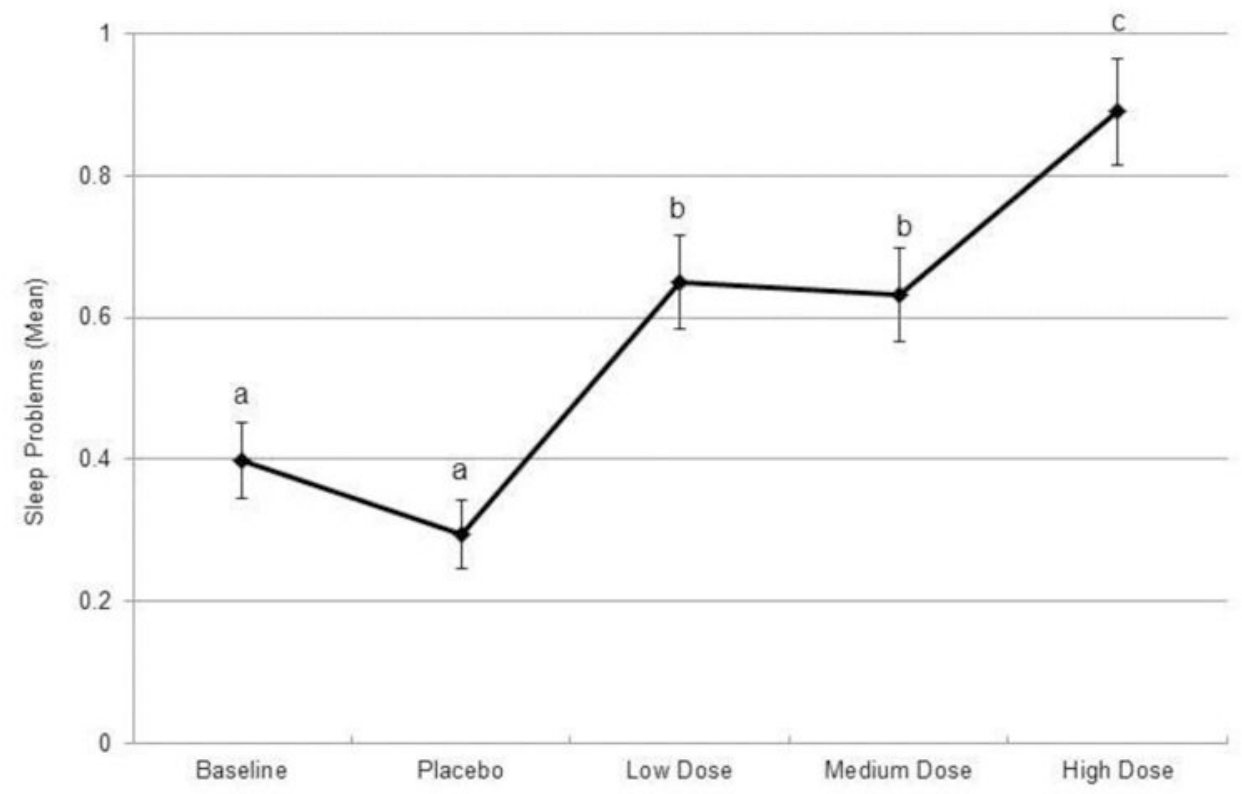

Figure 1. Effect of methylphenidate (MPH) dose on sleep problems in children with ADHD Note. Time-points with different superscripts differ significantly $(p<.05)$ in sleep problem severity such that $\mathrm{a}<\mathrm{b}<\mathrm{c}$, while time-points with the same superscript did not differ $(p>$ $0.05)$. 

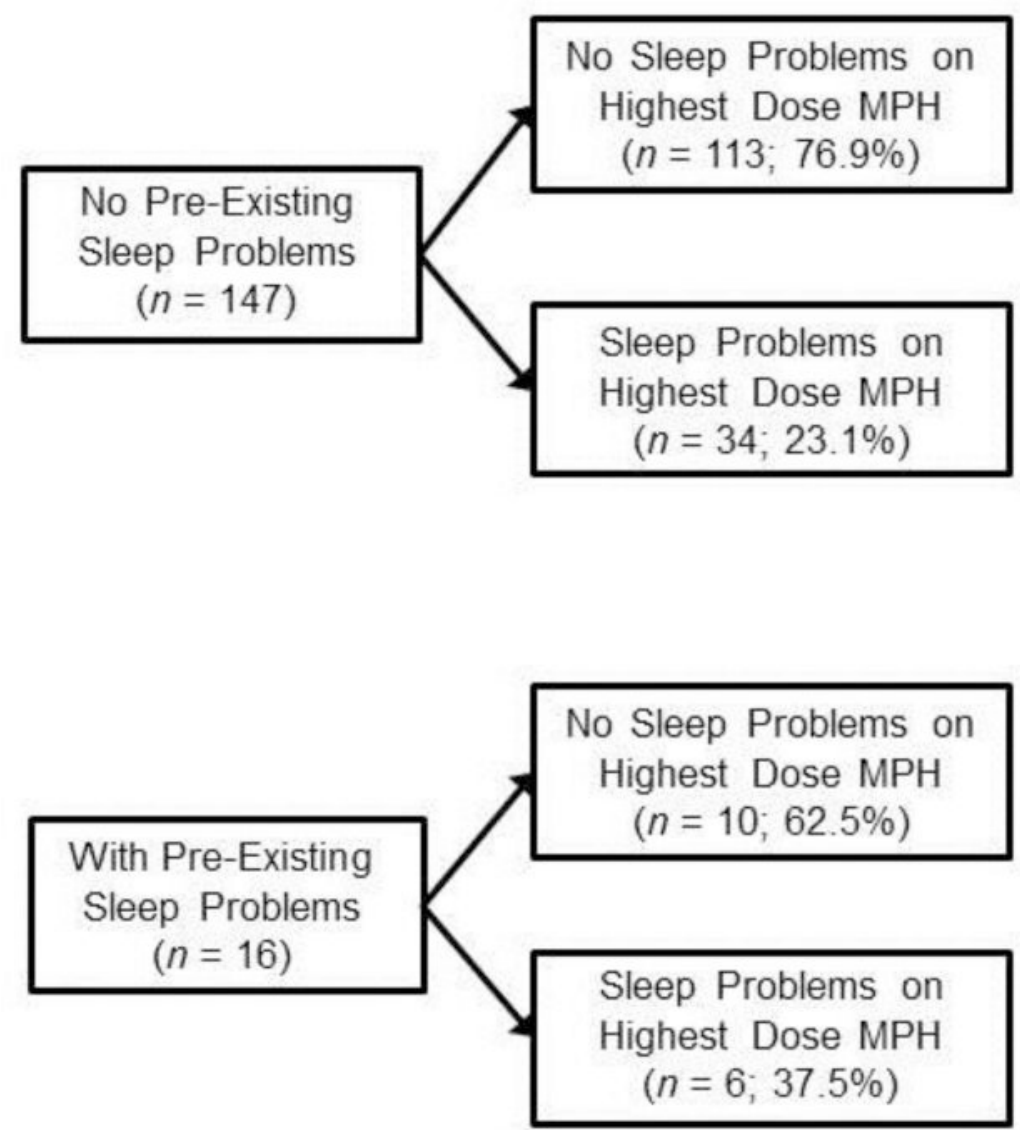

Figure 2. Number of Children with and without Pre-Existing Sleep Problems and Sleep Problems at the High Dose of MPH

Note. The presence or absence of sleep problems was determined based on parent ratings of sleep, with "none" or "mild" sleep problems classified as sleep problems absent and "moderate" or "severe" sleep problems classified as sleep problems present. MPH = methylphenidate. 


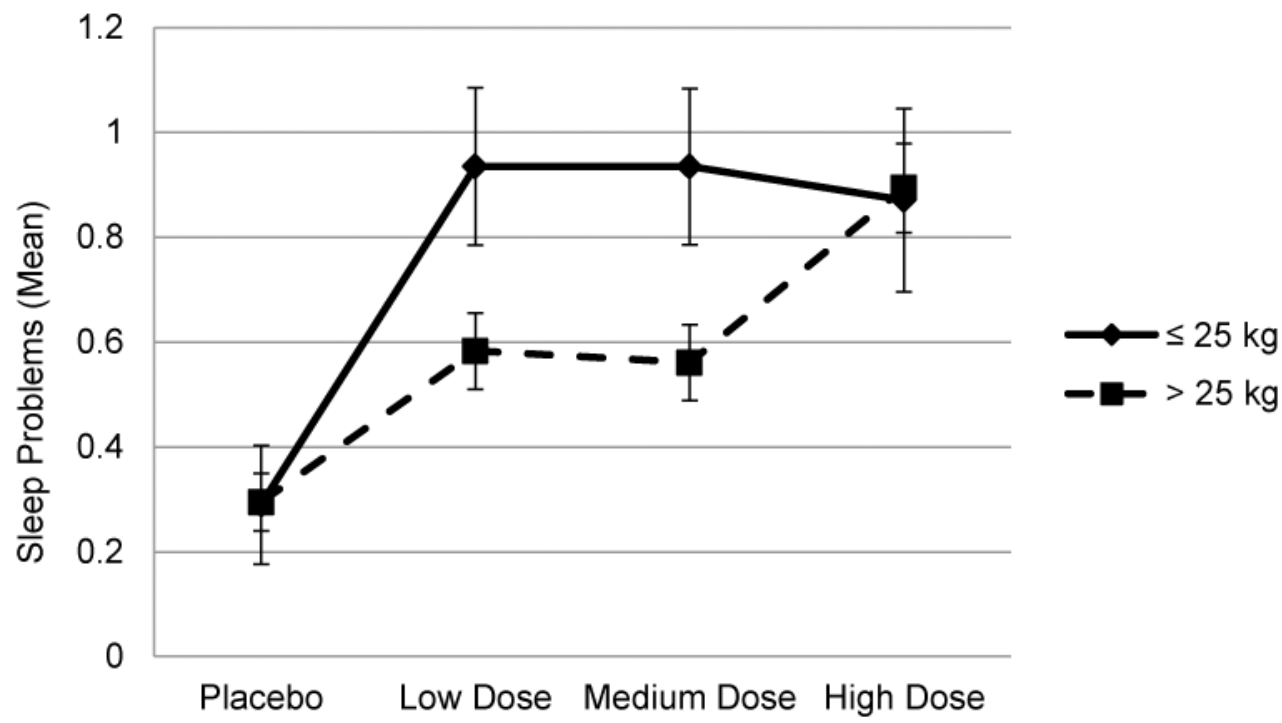

Figure 3. Interaction of weight and methylphenidate (MPH) dose on the sleep problems in children with ADHD 


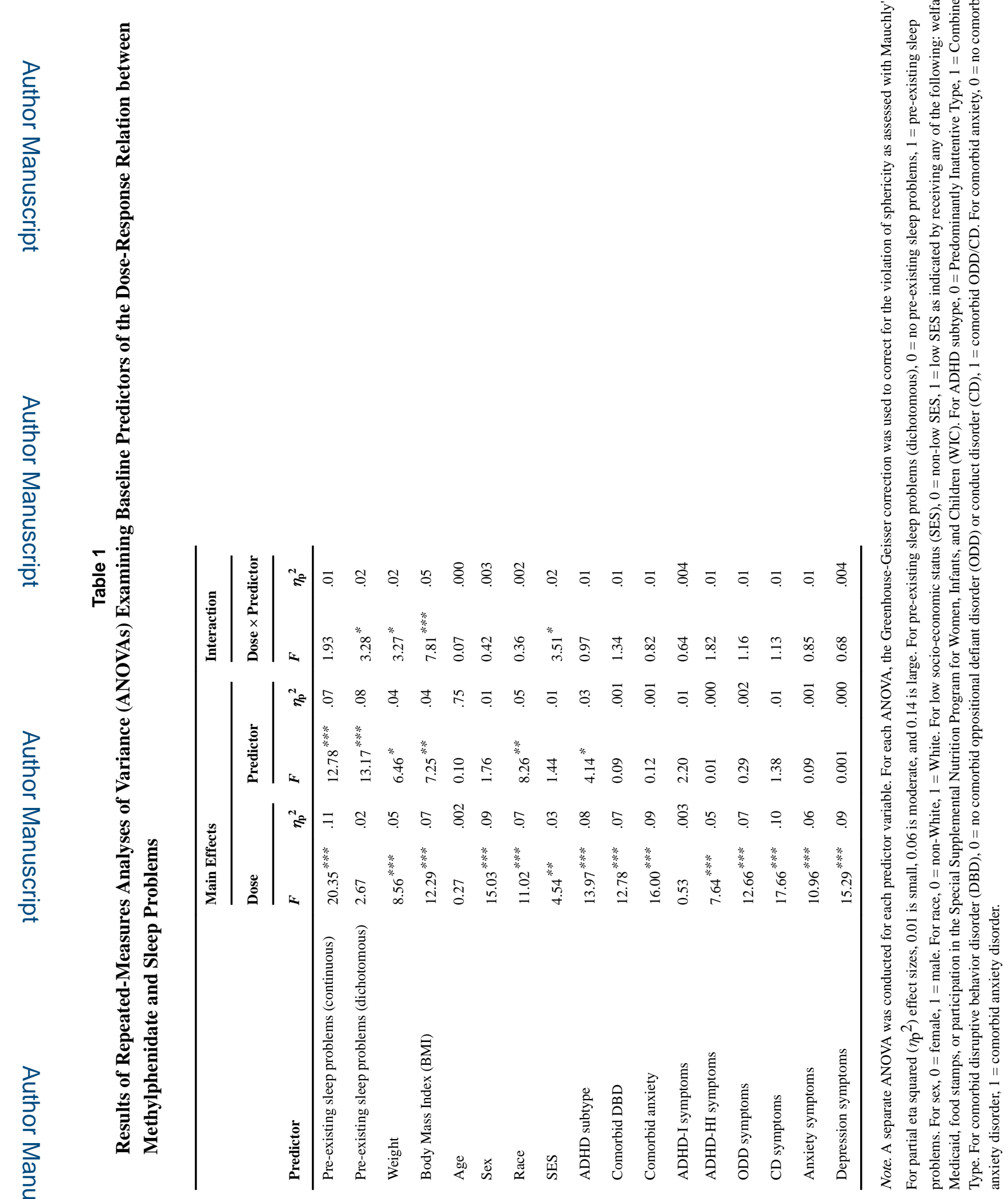




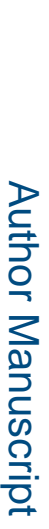

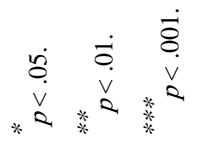

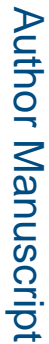

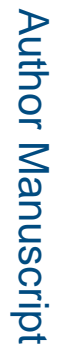

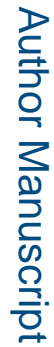

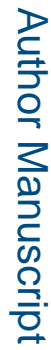

J Dev Behav Pediatr. Author manuscript; available in PMC 2017 June 01. 